\title{
Les implications du mariage précoce pour les politiques de $\mathrm{VIH} /$ SIDA
}

Judith Bruce

Population Council

Shelley Clark

Follow this and additional works at: https://knowledgecommons.popcouncil.org/departments_sbsr-pgy

Part of the Demography, Population, and Ecology Commons, Family, Life Course, and Society Commons, International Public Health Commons, and the Medicine and Health Commons How does access to this work benefit you? Let us know!

\section{Recommended Citation}

Bruce, Judith and Shelley Clark. 2004. "Les implications du mariage précoce pour les politiques de VIH/ SIDA." New York: Population Council. 


\title{
Les implications du mariage précoce pour les politiques de VIH/SIDA
}

Basé sur une recherche effectuée par

Judith Bruce

Directrice de la Division des Programmes Internationaux sur le Genre, la Famille et le Développement

Population Council

jbruce@popcouncil.org

\author{
Shelley Clark \\ Professeur Assistant \\ Harris Graduate School of Public Policy (Ecole Universitaire \\ Supérieure de Politique Publique) \\ Université de Chicago \\ sclark1@uchicago.edu
}

Ce dossier est basé sur un document contextuel préparé pour la Consultation Technique OMS/ FNUAP/ Population Council sur les Adolescentes Mariées, tenue à Genève en Suisse du 09 au 12 Décembre 2003 (le document final intitulé "Inclure les adolescentes mariées dans les politiques de santé reproductive des adolescents et de VIH/SIDA" par Judith Bruce et Shelley Clark est en voie d'achèvement et sera disponible sur le site www.popcouncil.org en Juin 2004). La Consultation a réuni des experts provenant des Nations Unies, des bailleurs de fonds et des agences non gouvernementales pour examiner les faits concernant la santé reproductive des adolescentes mariées, leur vulnérabilité face à l'infection du VIH, leurs inégalités sociales et économiques ainsi que leurs droits. Les rapports avec des initiatives politiques majeures, notamment la maternité sans risque, le VIH, la santé sexuelle et reproductive des adolescents et leurs droits à la reproduction ont été examinés et les résultats émergeant des programmes encore relativement rares en direction de cette population ont été l'objet de discussion. La réunion ainsi que les documents spécifiques ont été supportés en partie par un financement provenant de la Fondation Ford, de la Fondation Bill et Melinda Gates, de la Fondation John D. et Catherine T. MacArthur, du Population Council, du Département du Royaume Uni pour le Développement International, du FNUAP et de l'OMS. 


\section{UNFPA}

Le Fonds des Nations Unies d'Aide aux Populations (UNFPA) appuie les pays en développement, à leur demande, pour améliorer l'accès aux soins de santé reproductive et leurs qualités particulièrement le planification familiale, la maternité sans risque et la prévention des infections sexuellement transmises, notamment le VIH/SIDA. Les priorités comportent la protection des jeunes gens, la réponse aux urgences, et l'assurance d'une offre suffisante de condom et d'autres produits essentiels. Le Fonds participe également à la promotion des droits de la femme et donne son appui à la collecte et à l'analyse des données pour aider les pays à atteindre un développement durable. Environ un quart de toute l'assistance aux populations provenant des pays donateurs à ceux en développement est canalisé à travers le UNFPA qui travaille avec de nombreux gouvernements, organisations non gouvernementales et partenaires des Nations Unies.

\section{Population Council}

Le Population Council est une organisation non gouvernementale internationale à but non lucratif qui cherche à améliorer le bien être et la santé reproductive des générations actuelles et futures à travers le monde, et à contribuer pour atteindre un équilibre humain équitable et durable entre les peuples et les ressources. Le Council effectue des recherches dans les domaines de la biomédecine, des sciences sociales et de la santé publique, et aide également à renforcer les capacités de recherche dans les pays en développement. Créé en 1952, le Council est gouverné par un Conseil d'Administration international. Son siège social de New York soutient un réseau global de bureaux régionaux et nationaux.

Ce document n'est pas une publication formelle de l'Organisation Mondiale de la Santé ou du Fonds des Nations Unies d'Aide aux Populations. Les opinions exprimées ici sont celles de leurs auteurs et ne reflètent pas nécessairement les positions de l'OMS ou du FNUAP.

Auteur suggéré: Bruce, Judith and Shelley Clark. 2004. "The implications of early marriage for HIV/AIDS policy,” brief based on background paper prepared for the WHO/UNFPA/Population Council Technical Consultation on Married Adolescents. New York: Population Council. 


\section{INTRODUCTION}

Au cours de la dernière décennie, l'attention des politiques s'est focalisée sur les questions de santé reproductive et de développement social des adolescents. Pendant cette même période, les femmes en sont arrivées à représenter la moitié des personnes infectées par le VIH/SIDA. Dans certaines parties du monde, plus remarquablement en Afrique subsaharienne, les taux de prévalence du VIH parmi les jeunes femmes âgées de 15 à 24 ans l'emportent sur ceux des hommes du même groupe d'âge par deux à huit fois ${ }^{1}$. De conséquence considérable, mais encore largement ignoré, est le fait que la majorité des filles sexuellement actives de 15 à 19 ans dans les pays en développement sont mariées ${ }^{2}$, et ces adolescentes mariées tendent à avoir des taux d'infection par le VIH plus élevés que leurs pairs célibataires sexuellement actives ${ }^{3}$. Ainsi, les adolescentes mariées représentent non seulement une fraction importante des adolescentes exposées au risque, mais elles connaissent aussi certains des taux de prévalence de VIH les plus élevés de tous les groupes.

Néanmoins, les adolescentes mariées ont été marginales dans les politiques et programmes de VIH/SIDA des adolescents, et n'ont pas été les sujets principaux des programmes en direction des femmes adultes mariées ${ }^{4}$. Il est temps - en vérité il est tard - pour accorder une attention considérablement plus grande au rôle que joue le mariage précoce en ce qu'il expose potentiellement les filles et jeunes femmes à de graves risques de santé reproductive, notamment le VIH. Protéger ces jeunes femmes peut non seulement servir à contribuer à la prévention de la propagation de la maladie à partir des groupes à "haut risque" vers la population générale au sein de leur propre génération, mais aussi vers la génération suivante en réduisant la transmission de la mère à l'enfant parmi ce groupe en âge de procréation très intensive ${ }^{5}$.

\section{OMISSION TRADITIONNELLE DES ADOLESCENTES MARIEES}

Le mariage précoce et les besoins des adolescentes mariées ont traditionnellement été négligés. Le programme des politiques en direction des adolescents, dans leur brève histoire, a été contenu dans le cadre des priorités et expériences culturelles des pays développés, où les proportions d'adolescentes mariées sont relativement faibles. Etant donné leur petit nombre dans ces pays, les besoins et situations des adolescentes mariées ont été, au mieux, une considération mineure. En revanche, l'expérience d'initiation sexuelle des adolescentes célibataires - souvent des élèves - leurs comportements de prise de risque et, plus récemment, leurs environnements sociaux ont été les sujets principaux d'interventions de recherche et de politique à la fois.

\footnotetext{
${ }^{1}$ Laga, M., B. Schärtlander, E. Pisani, P.S. Sow, et M. Caraël. 2001. “To stem HIV in Africa, prevent transmission to young women,” AIDS 15(7): 931-934; et ONUSIDA. 2000. Report on the Global HIV/AIDS Epidemic. Washington, DC: ONUSIDA

${ }^{2}$ Ceci est basé sur une analyse de données d'Enquêtes Démographiques et de Santé (EDS) provenant de 26 pays. Elle exclut la Chine, où le mariage est typiquement tardif et où les données font défauts. Pour plus de détails voir le document d'où ce résumé a été extrait (Bruce, Judith et Shelley Clarke. 2004. "Including married adolescents in adolescent reproductive health and HIV/AIDS policy," background paper. New York: Population Council.).

${ }^{3}$ Glynn, J.R., M. Caraël, B. Auvert, M. Kahindo, J. Chege, R. Musonda, F. Kaona, et A. Buvé pour le Groupe d'Etude sur l'Hétérogénéité des épidémies de VIH dans les Villes Africaines. 2001. "Why do young women have a much higher prevalence of HIV than young men? A study in Kisumu, Kenya et Ndola, Zambie," AIDS 15(suppl 4): S51-S60; and Kelly, R.J., R.H. Gray, N.K. Sewankambo, D. Serwadda, F. Wabwire-Mangen, T. Lutalo, et M.J. Wawer. 2003.

"Age differences in sexual partners and risk of HIV-1 infection in rural Uganda," Journal of Acquired Immune Deficiency Syndromes 32(4): 446-451.

${ }^{4}$ Parce que le mariage précoce est un phénomène largement féminin, les filles ont des taux de VIH plus élevés dans la plupart des pays où la transmission hétérosexuelle est dominante, et peu de données existent sur les garçons. Ce document se concentre sur la situation des filles mariées.

${ }^{5}$ La procréation est attendue aussitôt après le mariage. En effet, tandis que l'âge au mariage a généralement augmenté, le nombre moyen de mois entre le mariage et la première naissance a baissé dans toutes les régions. Source: Mensch, Barbara. 2003. "Trends in the timing of first marriage," document présenté à la Consultation Technique OMS/FNUAP/Population Council sur les Adolescents Mariés, OMS, Genève, 9-12 Décembre.
} 
Juridiquement, les adolescentes mariées ont été laissées de côté. La Convention sur les Droits de l'Enfant (CDE) offre une définition transculturelle extrêmement utile de l'"enfance" (jusqu'à l'âge de 18 ans), ainsi qu'une vision détaillée des besoins et droits de l'enfant et de ses capacités en développement ; pour autant, elle permet aux pays d'appliquer ces droits et protections uniquement aux célibataires. La CDE permet aux pays signataires de déterminer si le mariage retire les filles (qui forment l'écrasante majorité des enfants mariées) ${ }^{6}$ et les garçons de l'espace protégé de l'enfance. En effet, certains pays auraient pu ne pas signer la CDE sans la dispense potentielle à la protection des droits de l'enfant pour les filles et femmes mariées. Cette construction juridique reflète et est justifiée par une norme culturelle longtemps existante qui veut que le mariage, quel que soit l'âge, confère le statut d'adulte. Une troisième raison étroitement liée à l'invisibilité des besoins des adolescentes mariées est l'idée qu'une fille mariée est "prise en charge" et qu'elle est passée de la "protection" de ses parents de naissance à celle de son mari.

Ces influences historiques, juridiques et culturelles, tout en étant compréhensibles, ont conduit au déni collectif de la survenance encore vivace et répandue du mariage des enfants. Sur les 331 millions de filles à présent âgées de 10 à 19 ans et vivant dans les pays du monde en développement, à l'exception de la Chine, 163 millions seront mariées au plus tard à leur vingtième anniversaire, si la tendance actuelle continue. Au cours des dix prochaines années, plus de 100 millions de filles dans ces pays seront mariées avant leur dix huitième anniversaire ${ }^{7}$.

Les stratégies initiales réussies dans la protection contre la transmission du VIH ont pris en charge les besoins de la première vague d'infection. Aux Etats-Unis, l'épidémie a été signalée d'abord parmi des hommes relativement riches, bien éduqués et qui avaient des rapports sexuels avec des hommes. En Afrique subsaharienne, la première vague d'infection a été trouvée parmi des "hommes riches qui pouvaient se permettre de voyager, d'avoir des partenaires sexuels multiples et de payer pour des relations sexuelles" ${ }^{8}$.

De manière croissante, cependant, l'épidémie dans toutes les régions progresse rapidement chez les pauvres et ceux qui sont dépourvus de pouvoir de négociation des termes de la sexualité, et par conséquent, choisit de plus en plus des jeunes gens, particulièrement des filles et jeunes femmes. Les stratégies qui se sont avérées efficaces pour les groupes antécédents de population vulnérable ne seront pas nécessairement des choix appropriés ou faisables pour des jeunes femmes impuissantes devant la pression de tomber enceintes.

\section{POURQUOI LES ADOLESCENTES MARIEES SONT-ELLES VULNERABLES?}

Les filles mariées avant l'âge de 18 ans sont en présence de risque considérable de VIH. Traverser le seuil du mariage augmente énormément l'exposition sexuelle par le biais de rapports sexuels non protégés qui ont lieu souvent avec un partenaire plus âgé qui, du fait de son âge, présente un risque élevé d'être VIH positif. Cette augmentation dramatique dans la fréquence des rapports non protégés est poussée non seulement par l'implication d'infidélité ou de suspicion associée avec certaines formes

\footnotetext{
${ }^{6}$ Dans presque tous les pays, les filles âgées de 15 à 19 ans ont deux fois plus de chance d'être mariées que les garçons; parfois la probabilité est beaucoup plus élevée. Par exemple au Brésil, la probabilité de mariage pour les filles est cinq fois plus élevée tandis qu'en Indonésie, elle l'est de 5,7 fois. Au Kenya, les filles ont 21 fois plus de chance de se marier que les garçons du même âge. Source: Nations Unies. 2000. World Marriage Patterns. New York: Division des Nations Unies pour la Population, Département des Affaires Economiques et Sociales

${ }^{7}$ Analyse de données d'EDS de Population Council, avec des remerciements spéciaux à Carey Meyers et Brian Pence

${ }^{8}$ Kiragu, Karusa. 2001. "Youth and HIV/AIDS: Can we avoid catastrophe?" Population Reports series L, no. 12, Fall. Baltimore:

Johns Hopkins University Bloomberg School of Public Health, Population Information Program, p. 5
} 
contraceptives comme le condom, mais souvent aussi par un fort désir de tomber enceinte. Nous avons examiné les données collectées dans les Enquêtes Démographiques et de Santé (EDS) provenant de 31 pays pour déterminer l'exposition potentielle des adolescentes aux risques du VIH à travers le mariage précoce (voir tableau page 4). Nous avons trouvé qu'en moyenne $80 \%$ des rencontres sexuelles non protégées parmi les adolescentes survenaient dans le cadre du mariage. Dans six pays (Bangladesh, Egypte, Inde, Indonésie, Sénégal et Turquie) nous ne détenons pas de données sur les comportements sexuels des adolescentes et donc ce chiffre se rapporte seulement aux 25 autres pays. Etant donné que selon les hypothèses, l'activité sexuelle chez les adolescentes célibataires dans les pays omis est faible, l'estimation globale des rapports sexuels dans le cadre du mariage au niveau de tous les 31 pays est probablement supérieure à $80 \%$.

En outre, l'augmentation du nombre de jeunes femmes infectées par le VIH a conduit certains décideurs et chercheurs à la conclusion que des importants écarts d'âge chez les partenaires sexuels laissent les adolescentes particulièrement vulnérables aux infections. Les risques associés aux grandes différences d'âge sont le plus souvent reconnus dans les relations entre des jeunes filles célibataires et des «sugar daddies » plus vieux. Bien que moins souvent soulignés, les importants écarts d'âge entre maris et jeunes mariées (femmes mariées avant l'âge de 20 ans) sont courants, variant entre 4,7 années au Guatemala à 14,1 ans en Guinée (Colonne 4 du tableau). En effet, plus la mariée est jeune au moment du mariage, plus important est l'écart d'âge avec son époux. Les maris sont en moyenne non seulement plus âgés que les petits amis, mais également, ils sont plus aptes à être infectés. Clark (2004) a calculé que dans Kisimu au Kenya, 30\% des partenaires masculins des adolescentes mariées étaient infectés par le VIH, tandis que seulement $11,5 \%$ des partenaires des filles célibataires étaient VIH positif. De même, à Ndola, en Zambie, 31,6\% des partenaires des filles mariées, comparés à 16,8\% des petits amis des filles célibataires ont été avérés porteurs du $\mathrm{VIH}^{9}$. Il y a une préoccupation croissante au sujet des grands écarts d'âge entre les partenaires sexuels dans les politiques nationales de SIDA. Toutefois, ces politiques manquent souvent de reconnaître le rôle du mariage dans la création et l'ancrage de tels importants écarts d'âge.

Enfin, dans la plupart des pays, les filles mariées font état de la solitude du mariage, de leur séparation d'avec leurs amis et familles, de la diminution de leur mobilité sociale et géographique, de leur accès limité à l'information, à l'école et la participation communautaire. Par exemple, la Colonne 5 souligne les faibles taux de participation communautaire chez les adolescentes mariées. L'isolement social est une perte en soi et elle est de plus en plus identifié comme un facteur prédisposant aux risques du VIH, car il sape les avantages de la « cohésion sociale ». Les contacts et réseaux sociaux sont de plus en plus reconnus comme une dimension vitale pour transmettre les informations et soutenir un changement de comportement. De même, les médias et les écoles sont de plus en plus engagés pour transmettre des messages de prévention du VIH et soutenir des programmes de VIH.

Non seulement les adolescentes mariées sont souvent isolées au sein de leur nouveau foyer et également du soutien extérieur public comme privé, mais encore, leurs besoins n'ont pas été considérés prioritaires ni même parfois pris en compte dans les initiatives de santé reproductive. Par ailleurs, un bon nombre des politiques et messages de VIH/SIDA les plus communs ne sont pas appropriés pour elles.

\footnotetext{
${ }^{9}$ Clark, Shelley. 2004. "Early marriage and HIV risks in sub-Saharan Africa," Harris School Working Paper Series no. 04.06. Chicago: University of Chicago
} 
Quatre principaux types de programmes de santé reproductive des adolescents consomment la grande majorité des ressources de santé reproductive des adolescents : les programmes d'éducation à la vie familiale qui comportent l'éducation sur le VIH/SIDA, les centres de jeunes, l'éducation par les pairs comme stratégie principale de communication, et les services conviviaux de santé pour les jeunes. Plus récemment, des efforts ont été faits pour engager les jeunes à travers divers médias. Souvent les filles mariées n'ont reçu aucune éducation scolaire ou ont quitté l'école assez tôt ; par conséquent ne peuvent pas recevoir les avantages de l'éducation à la vie familiale. Les filles en général, et certainement les filles mariées ne sont pas servies ou sont moins bien servies dans les centres de jeunes que les mâles ${ }^{10}$. Ainsi que l'indiquent les données ci-dessus sur les adolescentes mariées, ces dernières ont des schémas distinctifs de mobilité sociale et des réseaux sociaux plus restreints que les filles célibataires, les plaçant, sans doute, hors de la portée des programmes conventionnels de pair-à-pair. Enfin, et paradoxalement, les services de santé conviviaux pour les jeunes, qui étaient voulus comme un moyen principal d'améliorer la santé reproductive des adolescents, sont pour une grande part des services de contraception avec certaines informations sur les infections sexuellement transmises (IST) et le VIH, le counseling, y compris le dépistage et, le traitement lorsque disponible. Les programmes de santé reproductive des adolescents jusqu'à présent, accordent une faible attention à la préparation au mariage et souvent excluent explicitement les soins prénatals, d'accouchement et de post-partum comme des services clés.

A part les services de santé sexuelle et reproductive des adolescents, il y a d'autres voies clés pour atteindre les adolescentes mariées par le biais d'initiatives de maternité sans risque ou de services de santé maternelle. Les adolescentes, cependant, peuvent être servies insuffisamment par ces programmes prénatals et de post-partum, étant donné les risques élevés (sociaux et cliniques combinés) de la première naissance pour les plus jeunes mères ${ }^{11}$. Par ailleurs, bon nombre des services offerts, comme la contraception et la stérilisation, ne sont pas demandés par des jeunes femmes récemment mariées.

De même, les stratégies préconisées actuellement pour la protection contre le VIH/SIDA et la réduction du risque ont été :

- l'abstinence de l'activité sexuelle,

- la réduction de la fréquence sexuelle,

- le changement de partenaire sexuel (au profit d'un partenaire moins risqué),

- l'utilisation du condom (masculin ou féminin), et

- les relations mutuellement monogames avec un partenaire non infecté.

Aucune de ces stratégies à l'exception de la dernière (les relations mutuellement monogames avec un partenaire non infecté) n'offre un choix faisable pour les filles/femmes nouvellement mariées qui subissent la pression de tomber enceinte avec des maris plus puissants et/ou âgés.

\section{EXERCICES POUR GUIDER LES EVALUATIONS SPECIFIQUES DES RISQUES DE VIH POUR LES ADOLESCENTES MARIEES A UN PAYS}

\footnotetext{
${ }^{10}$ Erulkar, Annabel. 2003. "Examining the gender dimensions of popular adolescent programs: What they could offer adolescent girls and boys," dans Adolescent and Youth Sexual and Reproductive Health: Charting Directions for a Second Generation of Programming, document de fond pour un atelier du FNUAP en collaboration avec le Population Council. New York: Population Council

${ }^{11}$ Miller, Suellen et Felicia Lester. 2003. "Re-orienting information, social support and services for the youngest mothers," document présenté à la Consultation Technique OMS/FNUAP/Population Council sur les Adolescents Mariés, OMS, Genève, 9-12 Décembre
} 
Les gouvernements peuvent déterminer combien leurs populations d'adolescentes mariées sont vulnérables au VIH ainsi que l'ampleur du problème en terme de propagation de l'épidémie à la «population générale », voulant signifier habituellement les hommes et femmes mariés. Ces analyses se proposent d'aider à identifier quelles interventions de VIH et de santé reproductive sont le plus urgemment requises pour les adolescentes mariées et/ou les femmes mariées en général. Il y a 4 parties essentielles dans cette analyse.

\section{Estimer les niveaux et la distribution du VIH/SIDA dans la population actuelle}

L'estimation de la prévalence spécifique à l'âge et au sexe et, lorsque possible, de l'incidence parmi les différentes populations, constitue le premier pas pour évaluer quel rôle, le cas échéant, le mariage précoce ou le mariage en soi jouera soit pour contrer la vague du VIH ou servir comme pont vers la population générale. Il est important, quoique souvent difficile, de collecter des données sur la prévalence à partir de groupes généralement considérés comme faiblement exposés aux risques, comme les jeunes mariées monogames ${ }^{12}$.

Mesures de l'exposition potentielle des adolescentes aux risques de VIH par le mariage précoce, par pays (données d'EDS).

\begin{tabular}{|c|c|c|c|c|c|c|c|}
\hline & & Colonne 1 & Colonne 2 & Colonne 3 & Colonne 4 & Colonne 5 & \\
\hline Pays & Année & $\begin{array}{c}\text { Pourcentage } \\
\text { de mariées à } \\
18^{\mathrm{a}} \text { ans au } \\
\text { plus tard }\end{array}$ & $\begin{array}{l}\text { Pourcentage } \\
\text { de mariées } \\
\text { ayant eu une } \\
\text { relation } \\
\text { sexuelle non } \\
\text { protégée } \\
\text { durant la } \\
\text { semaine } \\
\text { passée } \\
\end{array}$ & $\begin{array}{l}\text { Risque relatif } \\
\text { d'avoir eu } \\
\text { des rapports } \\
\text { non protégés } \\
\text { la semaine } \\
\text { passée (prob. } \\
\text { mariées/prob. } \\
\text { célibataires }\end{array}$ & $\begin{array}{c}\text { Différence } \\
\text { d'âge moyen } \\
\text { entre époux } \\
\text { (mariées < à } \\
20 \text { ans) }\end{array}$ & $\begin{array}{c}\text { Pourcentage } \\
\text { d'adolescentes } \\
\text { élèves mariées }\end{array}$ & $\begin{array}{c}\text { Nombre } \\
\text { d'adolescentes } \\
\text { (âgées de } 15 \text { à } \\
19 \text { ans) }\end{array}$ \\
\hline \multicolumn{8}{|l|}{$\begin{array}{l}\text { Afrique } \\
\text { Australe et } \\
\text { de l'Est }\end{array}$} \\
\hline Ethiopie & 2000 & 49.1 & 97.9 & 141.6 & 8,7 & N/A & 3.366 \\
\hline Kenya & 1998 & 24.6 & 72.3 & 14.1 & 7.9 & 2.0 & 1827 \\
\hline Malawi & 2000 & 46.9 & 92.6 & 24.3 & 5.7 & N/A & 2788 \\
\hline Mozambique & 1997 & 56.6 & 82.0 & 5.4 & 7.7 & 2.2 & 1816 \\
\hline Rwanda & 2000 & 19.5 & 97.2 & 491.4 & 6.3 & N/A & 2713 \\
\hline $\begin{array}{l}\text { Afrique du } \\
\text { Sud }\end{array}$ & 1998 & 7.9 & 12.6 & 4.3 & 6.2 & 37.7 & 2357 \\
\hline Tanzanie & 1999 & 39.3 & 77.0 & 9.7 & $\mathrm{~N} / \mathrm{A}$ & 0.0 & 909 \\
\hline Ouganda & 1995 & 54.1 & 96.3 & 27.7 & N/A & 1.2 & 1578 \\
\hline Zambie & $2001 / 02$ & 42.1 & 81.7 & 13.4 & 6.7 & N/A & 1756 \\
\hline Zimbabwe & 1999 & 28.7 & 94.7 & 63.9 & 8.3 & N/A & 1454 \\
\hline \multicolumn{8}{|l|}{$\begin{array}{l}\text { Afrique de } \\
\text { l'Ouest }\end{array}$} \\
\hline Bénin & 2001 & 36.7 & 68.1 & 7.0 & 9.5 & $\mathrm{~N} / \mathrm{A}$ & 1216 \\
\hline Burkina Faso & $1998 / 99$ & 62.3 & 86.0 & 11.9 & 11.5 & 0.2 & 1461 \\
\hline Cameroun & 1998 & 43.4 & 69.9 & 4.4 & 11.1 & 6.0 & 1269 \\
\hline Gabon & 2000 & 33.6 & 53.2 & 4.9 & 8.5 & 38.1 & 1552 \\
\hline Ghana & 1998 & 35.5 & 49.0 & 6.0 & 8.5 & 3.5 & 866 \\
\hline Guinée & 1999 & 64.5 & 84.0 & 6.5 & 14.1 & 2.8 & 1317 \\
\hline Mali & 2001 & 65.4 & 89.6 & 9.6 & 12.1 & N/A & 2477 \\
\hline Nigeria & 1999 & 39.6 & 89.3 & 45.4 & 11.9 & 2.3 & 3365 \\
\hline
\end{tabular}

\footnotetext{
${ }^{12}$ Bien que certaines filles mariées soient prises à partir de données d'observatoire recueillies auprès de cliniques prénatales, il n’y a pas eu d'enquête spécifique de jeunes mariées.
} 


\begin{tabular}{|c|c|c|c|c|c|c|c|}
\hline Sénégal & 1999 & 36.1 & N/A & N/A & $\mathrm{N} / \mathrm{A}$ & N/A & 1935 \\
\hline \multicolumn{8}{|l|}{$\begin{array}{l}\text { Amérique } \\
\text { Latine et } \\
\text { Caraïbes }\end{array}$} \\
\hline Brésil & 1996 & 23.7 & 71.7 & 15.3 & 5.7 & 11.1 & 2453 \\
\hline Colombie & 2000 & 21.4 & 77.6 & 19.8 & 6.2 & N/A & 2192 \\
\hline $\begin{array}{l}\text { République } \\
\text { Dominicaine }\end{array}$ & 1999 & 31.0 & 95.6 & 89.4 & 7.2 & 38.5 & 249 \\
\hline Guatemala & $1998 / 99$ & 34.3 & 100.0 & N/A & 4.7 & 2.9 & 1366 \\
\hline Haïti & 2000 & 24.1 & 82.6 & 23.8 & 7.1 & N/A & 2367 \\
\hline Nicaragua & 2001 & 50.3 & 97.3 & 112.3 & 5.5 & N/A & 3074 \\
\hline Pérou & 2000 & 18.7 & 81.2 & 37.2 & 4.8 & N/A & 5679 \\
\hline \multicolumn{8}{|l|}{ Asie } \\
\hline Bangladesh & $1999 / 2000$ & 65.3 & $\mathrm{~N} / \mathrm{A}$ & N/A & 9.5 & N/A & 1571 \\
\hline Inde & $1998 / 99$ & 47.6 & N/A & $\mathrm{N} / \mathrm{A}$ & 6.3 & N/A & 6888 \\
\hline Indonésie & 1997 & 29.6 & N/A & N/A & $\mathrm{N} / \mathrm{A}$ & 1.3 & 1066 \\
\hline \multicolumn{8}{|l|}{$\begin{array}{l}\text { Moyen } \\
\text { Orient }\end{array}$} \\
\hline Egypte & 2000 & 19.5 & N/A & N/A & 7.9 & N/A & 579 \\
\hline Turquie & 1999 & 23.0 & N/A & N/A & 5.2 & 0.5 & 1759 \\
\hline
\end{tabular}

Déterminer la prévalence du mariage précoce pour le pays tout entier et pour des sous populations spécifiques

La prévalence du mariage précoce varie énormément par pays (e.g. Colonne 1) ou dans un pays donné parmi des situations culturelles ou géographiques spécifiques. Par exemple, si l'âge moyen au mariage sur l'étendue de la nation est un important indicateur, de nombreux pays ont cependant, des âges au mariage très bas dans certaines parties spécifiques du pays.

\section{Déterminer l'ampleur des risques de VIH accompagnant le mariage précoce}

Pour déterminer la mesure où le mariage marque une augmentation brutale du risque de VIH, deux principales mesures devraient être considérées : le pourcentage d'activités sexuelles non protégées survenant dans le mariage par groupe d'âge (Colonne 2) et la différence d'âge moyenne entre les jeunes mariées (c'est-à-dire moins de 20 ans) et leurs époux (Colonne 4). Ainsi qu'il apparaît dans la discussion ci-dessus, le différentiel d'âge entre les partenaires peut servir de procuration non seulement pour le pouvoir relatif dans la relation, mais également la probabilité d'infection parmi le plus vieux des époux, typiquement le mari.

Evaluer la disponibilité des programmes et services et l'accès vers ceux-ci pour les femmes mariées, en particulier les femmes plus jeunes ou nouvellement mariées

Des analyses devraient être effectuées par les gouvernements et ONGs pour évaluer la portée des programmes qui ciblent actuellement ou pourraient cibler les adolescentes mariées. Sans considération pour le fait que ces programmes accordent ou non une priorité aux messages de VIH, tout programme social, économique ou sanitaire qui atteint les adolescentes mariées peut être perçu comme un véhicule important pour endiguer l'épidémie. Certains pays peuvent avoir des systèmes exceptionnels de contact avec les personnes qui sont fiancées, se marient ou sont des jeunes mariées. Si de telles procédures et infrastructures de services existent, elles peuvent se permettre des lieux de contact et d'intervention pratiques et culturellement acceptables. Dans d'autres pays, cependant, les couples récemment mariés peuvent avoir peu ou pas de contact avec les services publics comme l'éducation ou les soins sanitaires (voir Colonne 5 pour les faibles taux de scolarisation chez les adolescentes mariées). 


\section{ETUDES DE CAS}

Si l'ambition de ce résumé n'est pas d'identifier les besoins spécifiques pour tous les pays, sur la base de leurs profils de mariage et de $\mathrm{VIH}$, les quatre scénarios illustratifs ci-après semblent toutefois suggérer combien les évaluations qui précédent peuvent être utile pour décrire l'importance d'atteindre les femmes mariées, en particulier les adolescentes mariées. Chaque pays doit évaluer ses propres besoins et tailler sa réaction pour convenir à son contexte unique.

Le Burkina Faso connaît des taux faibles mais croissants de VIH parmi les sous populations à haut risque. Le taux de prévalence est considérablement plus élevé chez les jeunes femmes âgées de 15 à 24 ans, avec des estimations variant de $7,8 \%$ à $11,7 \%$, que parmi les jeunes hommes $(3,2 \%-4,8 \%)^{13}$.

Le mariage modèle profondément les comportements sexuels des filles au Burkina Faso, étant donné que les deux tiers environ des femmes âgées de 20 à 24 ans étaient mariées avant l'âge de 18 ans. En effet, les contrastes entre les filles mariées et non mariées sont marqués, peut-être davantage que dans beaucoup de situations. Les filles mariées sont plus susceptibles d'avoir des rapports non protégés. En effet, les filles mariées sont douze fois plus aptes à signaler des rapports sexuels non protégés au cours de la semaine précédente que les filles non mariées (Colonne 3). Plus frappant, la différence moyenne d'âge entre les époux est presque de 12 ans, suggérant que les maris sont plus susceptibles d'avoir considérablement plus d'exposition sexuelle que leurs femmes avant le mariage et sont plus susceptibles d'être infectés. Etant donné ce profil, nous pourrions classer le Burkina Faso comme un pays où le mariage précoce pour les filles peut être un point d'entrée particulièrement vulnérable pour le VIH vers la population générale.

La République dominicaine peut aussi être caractérisée comme ayant des taux faibles mais croissants de VIH parmi des groupes spécifiques. Quelque 31\% des femmes ont été mariées avant l'âge minimum légal de 18 ans. Et bien que plus d'un quart des filles âgées de 15 à 19 ans soient sexuellement actives, presque $80 \%$ de ces dernières sont mariées. Les adolescentes mariées ont des taux beaucoup plus bas d'utilisation du condom que leurs homologues célibataires. Lorsque les adolescentes ont été interrogées sur leurs rencontres sexuelles de la semaine passée, il a été révélé que $96 \%$ au total des rencontres sexuelles non protégées survenaient dans le mariage. Parmi les filles actuellement mariées et âgées de 15 à 19 ans, la différence moyenne d'âge entre les partenaires est de 7,2 ans. Du fait de leur âge, les partenaires plus âgés sont plus susceptibles d'avoir eu des partenaires sexuels antérieurs. Enfin, les adolescentes mariées sont socialement plus isolées que leurs homologues célibataires. Bien que plus de 90\% des filles célibataires de 15 à 19 ans fréquentent actuellement l'école en République dominicaine, 3 filles mariées sur 5 ne sont ni écolières ni travailleuses. Ainsi, bien que la prévalence globale du VIH soit faible selon les normes internationales, la fréquence élevée des rapports non protégés survenant dans le mariage et la grande différence d'âge des conjoints, auxquelles s'ajoute l'isolement social des adolescentes mariées, font de ces dernières un important groupe à surveiller et protéger en République dominicaine $^{14}$.

L'Inde est un exemple de l'importance qui réside dans l'évaluation des caractéristiques de chaque pays (et parfois même des régions dans un pays). Comme en République dominicaine, la prévalence actuelle de VIH est relativement faible avec moins d'un pourcent de la population infectée (bien que les taux

\footnotetext{
${ }^{13}$ ONUSIDA. 2000. "Report on the global HIV/AIDS epidemic." Washington, DC: ONUSIDA.

${ }^{14}$ Goldberg, Rachel. 2003. "Structures of risk: Gender and HIV/AIDS in the Dominican Republic," thèse de Master,

Columbia University Mailman School of Public Health
} 
soient déjà deux fois plus élevés chez les femmes que chez les hommes). Malgré l'absence de données concluantes, environ 20 à $25 \%$ des garçons célibataires à l'école ou d'âge scolaire ont signalé avoir des rapports sexuels avant le mariage - souvent avec une travailleuse du sexe ${ }^{15}$. Les filles célibataires cependant, sont tenues de s'abstenir de toutes activités sexuelles. Le mariage précoce continue d'être courant avec $47,6 \%$ des femmes mariées avant 18 ans. Le mariage, par conséquent, est la voie principale aux rapports non protégés, et ainsi nous pouvons déduire que la transition au mariage et aux risques de VIH inhérents est particulièrement abrupte. Le mariage marque également une transition vers un plus grand isolement social pour beaucoup de femmes en Inde ${ }^{16}$. Au mariage, les filles quittent souvent leur foyer natal et emménagent fréquemment dans les familles de leur mari qui peuvent habiter dans une autre ville ou village. Malgré le fait que pour les femmes, les rapports non protégés surviennent dans le contexte du mariage, il y a des limites extrêmes dans les connaissances ou perceptions des risques du VIH parmi les femmes mariées. Ceci est vrai même parmi les femmes VIH positif monogames à Mumbai $^{17}$. Tandis que le VIH a frappé des populations à haut risque comme les travailleuses du sexe à Mumbai, il y a des signes avertissant que la deuxième vague sera parmi les femmes mariées et que ces dernières sont mal préparées à se protéger et à protéger leurs enfants.

En Zambie, le VIH/SIDA a déjà atteint des niveaux endémiques au sein de la population générale. Les faibles estimations des taux de prévalence de $\mathrm{VIH}$ indiquent que $10 \%$ des hommes de 15 à 24 ans et $26 \%$ des femmes du même groupe d'âge sont infectés. Le mariage précoce est fréquent en Zambie : 42\% des femmes ont été mariées avant 18 ans. Peut être le fait plus particulier pour la Zambie est que moins de la moitié (44\%) des adolescentes sexuellement actives sont mariées, suggérant des taux relativement élevés d'activité sexuelle avant le mariage. Cependant, et ceci est frappant, les adolescentes représentent une majorité évidente de celles ayant signalé des rapports non protégés au cours de la semaine passée (82\%), essentiellement en raison d'une plus grande fréquence des rapports dans le mariage plutôt que d'une diminution de l'utilisation du condom. Ainsi, comme il apparaît dans la Colonne 3, les filles mariées sont plus de 13 fois plus susceptibles que les célibataires d'avoir eu des rapports non protégés au cours de la semaine écoulée. En moyenne, les maris des filles mariées avant 20 ans sont plus âgés que leurs femmes de 6,7 ans. Si la Zambie a fait la promotion de plusieurs grands programmes d'éducation à la vie familiale et de vulgarisation chez les jeunes, généralement ces programmes n'atteignent pas l'importante proportion des adolescentes qui sont mariées. Ainsi, la Zambie représente les pays où la nécessité de mettre en œuvre des efforts spéciaux pour atteindre les adolescentes mariées avec des stratégies de protection est particulièrement pressante, et où la non prise en compte des risques pour les femmes mariées peut saper les programmes d'intervention de VIH existants.

\section{EFFORTS DE PREVENTION POUR MINIMISER LES RISQUES DE VIH PROVENANT DU MARIAGE}

Les chefs séculiers et religieux doivent jouer un important rôle pour la réussite des efforts visant à combattre la transmission du VIH dans le mariage. Une décision politique clé, que les chefs doivent prendre, est de déterminer si le report du mariage jusqu'à l'âge de 18 ans et la sensibilisation sur les

\footnotetext{
${ }^{15}$ Santhya, K.G. et Shireen Jejeebhoy. 2003. "Sexual and reproductive health needs of married adolescent girls," Economic and Political Weekly 38(41): 11 Octobre

${ }^{16}$ Elul, Batya. 2003. "Induced abortion in Rajasthan, India: Prevalence estimates from two quantitative methodologies," communication à partir d'un document de travail.

${ }^{17}$ Gangakhedkar, R.R., M.E. Bentley, A.D. Divekar, D. Gadkari, S.M. Mehendale, M.E. Shepherd, R.C. Bollinger, et T.C. Quinn. 1997. "Spread of HIV infection in married monogamous women in India," Journal of the American Medical Association 278(23): 2090-2092; et Newmann, S., P. Sarin, N. Kumarasamy, E. Amalraj, M. Rogers, P. Madhivanan, T. Flanigan, S. Cu-Uvin, S. McGarvey, K. Mayer, et S. Solomon. 2000. "Marriage, monogamy and HIV: A profile of HIV-infected women in south India," International Journal of STD \& AIDS 11(4): 250-253
} 
risques de transmission du VIH dans le mariage constitueront des éléments explicites de la politique de prévention du VIH. Sous réserve que la volonté politique existe, d'autres mesures politiques spécifiques pour minimiser les risques de contraction et dissémination du VIH à travers le mariage peuvent être identifiées et mises en œuvre. Nous avons organisé ces options politiques en ordre chronologique depuis la période précédant le mariage à celle suivant la première naissance. L'ordre ne représente pas cependant un classement selon l'importance, et nous sommes certains qu'il ne couvre pas la gamme totale des options.

\section{Options pré-maritales}

\section{Evaluer la base juridique pour éliminer les mariages avant l'âge/des enfants}

Dans la plupart des pays, l'âge minimum au mariage a été établi dans le cadre de la charte des droits dans une société de définir le statut «d'adulte» ou de majorité, et d'offrir des protections aux jeunes hommes et femmes, et aux garçons et filles. Dans cet esprit, les pays doivent examiner la logique et la justice de leurs lois, leur interprétation culturelle, les niveaux de conformité et les implications pour le risque de VIH si ses lois sont violées. Dans certains endroits, assurer la base juridique pour reporter l'âge du mariage à 18 ans ou au delà, nécessite une confrontation avec les droits parentaux en compétition. Dans certains endroits, des filles d'un âge aussi jeune que 12 ans peuvent être mariées avec le « consentement parental ». L'élaboration de telles lois, bien sûr, présume la situation de jeunes personnes cherchant à se marier contre la volonté de leurs parents. Ce qui est beaucoup plus vraisemblable, c'est l'exercice par les parents d'un contrôle absolu sur leurs enfants, principalement sur les vies de leurs filles en imposant le mariage précoce, au nom de raisons économiques ou culturelles comme la protection de la réputation de la famille.

\section{Développer des initiatives à base communautaire redéfinissant un âge acceptable de mariage et offrant des incitations aux parents et aux filles pour retarder le mariage ou l'âge légal}

Une considérable attention a été accordée au rôle que joue l'éducation dans le mariage plus tardif. La relation entre le travail et le mariage plus tardif a été l'objet de moins d'attention, mais il y a certaines indications que les filles qui travaillent pour un salaire ont tendance à se marier plus tard. En effet, dans certaines communautés, la simple possibilité de travailler pour une rémunération (ce qui requiert une éducation scolaire plus élevée) peut en soi jouer un rôle dans le report du mariage ${ }^{18}$.

Etant donné la rareté des exemples d'efforts à base communautaire pour promouvoir le mariage plus tardif, nous pouvons considérer la littérature sur les efforts visant à réduire l'ampleur et le degré des mutilations génitales féminines (MGF). Bien que de nombreux pays où les MGF sont pratiquées aient interdit celles-ci pendant des dizaines d'années, ces lois étaient inappliquées et souvent inconnues. Par ailleurs, les MGF - comme le mariage précoce - ont souvent été décrites, même défendues comme partie intégrante de la culture locale. Les efforts à base communautaire déployés pour mettre fin aux MGF combinent typiquement la dissémination d'informations exactes, la mobilisation des parents, personnes âgées et autres leaders dans la communauté, le recyclage des exciseuses et dans certains cas des actions judiciaires contre celles qui effectuent les MGF ou contre les parents ${ }^{19}$.

\footnotetext{
${ }^{18}$ Amin, Sajeda, Ian Diamond, Ruchira Tinaved, et Margaret Nubi. 1998. "Transition to adulthood of female factory workers in Bangladesh," Studies in Family Planning 29(2): 185-200

${ }^{19}$ Mohamud, Asha, Samson Radeny, Nancy Yinger, Zipporah Kittony, et Karin Ringheim. 2002. "Protecting and empowering girls: Confronting the roots of female genital cutting in Kenya," in Nicole Haberland and Diana Measham (eds.), Responding to Cairo: Case Studies of Changing Practice in Reproductive Health and Family Planning. New York: Population Council, pp. 434-458; Ismailly, Jumbe. 2003. "Three get 30 years after FGM victim dies," http://www.ippmedia.com, 11 Octobre; and Ghana News Agency. 2003. "Woman gets five-year jail term for performing FGM," http://www.ghanaweb.com, 24 Septembre
} 
De nouvelles initiatives pour rendre le mariage plus sûr peuvent s'appuyer directement sur les structures existantes de mobilisation contre les MGF. Bien que nous soyons vraisemblablement incapables de traiter efficacement ou acceptablement du mariage précoce, à moins qu'il n'y ait une mobilisation au niveau de la communauté, à partir d'un certain moment les gouvernements nationaux devront être prêts à rendre responsables, à travers des mesures légales, ceux qui font la promotion du mariage précoce ou les imposent aux enfants ${ }^{20}$.

\section{Souligner l'importance de conjoints d'âges corrects et sans risques}

S'il est vrai que le VIH ne peut pas être diagnostiqué à partir de l'apparence d'un individu, ceci ne veut pas dire que certaines caractéristiques ne sont pas associées avec une plus grande probabilité d'infection. Les parents et les adolescentes doivent être conscients des corrélations entre l'âge des prétendants, leurs expériences sexuelles antérieures et la probabilité d'une infection de VIH. Un des plus importants facteurs augmentant l'âge au mariage pour les hommes se trouve dans la nécessité de disposer de suffisamment de ressources afin de payer une dot souvent considérable. Les politiques conçues pour minimiser ou éliminer la pratique de la dot ou d'autres échanges économiques au moment du mariage peuvent contribuer à diminuer les différences d'âge entre les conjoints.

Juger si le mariage plus tardif augmentera-t-il vraisemblablement le nombre des adolescents non mariés sexuellement actifs et vulnérables

La réticence à promouvoir le mariage plus tardif dans le cadre de la prévention du VIH peut être fondée sur une croyance répandue que le mariage plus tardif augmente le nombre des adolescents célibataires et sexuellement actifs, une préoccupation appliquée principalement aux adolescentes. Nous avons donné ci-dessus des raisons pour réfuter les perceptions communes sur l'adolescent irresponsable, dangereux, sexuellement actif et célibataire, par opposition aux relations présumément sans risque, mutuellement monogames dans le mariage. En outre, il y a peu de faits pour étayer la crainte que les adolescentes qui retardent le mariage initieront les rapports sexuels avant le mariage ${ }^{21}$.

\section{La transition du mariage}

Dans beaucoup de cultures, le mariage est un processus plutôt qu'un événement discret ${ }^{22}$. En effet, la frontière formelle du mariage dans certaines sociétés n'est pas ce qui est important, mais plutôt il y a un processus social parallèle - rituels, coutumes et ainsi de suite - qui démarque l'éligibilité au mariage et la recherche active de la grossesse. Ce processus social (cérémonie avant mariage, célébration, coutumes et déclarations) peut fournir une série de points d'entrée pour la promotion d'informations sur la communication du couple, le dépistage et counseling volontaire, le condom, la santé sexuelle, le mariage moins risqué et la procréation - notamment garantir des enfants sains, exempts de maladie - pour les couples nouvellement fiancés.

\footnotetext{
${ }^{20} \mathrm{Au}$ Kenya, où le mariage forcé est illégal, l'Ecole Primaire de Filles de l'African Inland Church offre une éducation et un abri de qualité aux jeunes filles "rescapées " des mariages précoces. Source: "FAWE: Centering on excellence," Carnegie Reporter 1(3): Fall 2001, www.carnegie.org/reporter/03/eduafrica/fawe.html

${ }^{21}$ Par exemple, dans une récente étude de 120 enquêtes effectuée depuis 1990 dans 71 pays dans le cadre de l'EDS, nous voyons que si les proportions de jeunes filles signalant des expériences sexuelles pré maritales sont relativement constants, parmi les 15 pays d'Afrique subsaharienne ayant deux enquêtes ou plus pendant cette période, l'âge moyen au premier mariage a augmenté de 4 mois environ. Pour plus de détails, voir le document de fond d'où ce résumé est extrait (Bruce, Judith et Shelley Clarke. 2004. "Including married adolescents in adolescent reproductive health and HIV/AIDS policy,”. New York: Population Council.

${ }^{22}$ Bledsoe, Caroline H. et Gilles Pison (eds.). 1997. Nuptiality in Sub-Saharan Africa: Contemporary Anthropological and Demographic Perspectives. Oxford: Clarendon Press
} 


\section{Redéfinir la première année de mariage comme une zone de santé}

Dans de nombreuses situations, cela pourrait être valable de caractériser la première année de mariage comme une «zone de santé » de la part des professionnels de santé publique. Dans le contexte d'un nouveau mariage et la perspective d'enfants futurs, assurer la sécurité de la mère et de l'enfant à la fois peut devenir un argument de poids pour le changement de comportement ${ }^{23}$.

\section{Susciter des relations plus intimes et sereines entre les nouveaux conjoints}

Les plus jeunes mariées et leurs partenaires sont souvent non seulement isolés de leurs pairs particulièrement pour la mariée - mais ils sont aussi insérés dans de plus grandes structures familiales. La recherche sur les plus jeunes nouveaux parents indique un désir pour une plus grande intimité et davantage d'échanges d'information pour les deux côtés ${ }^{24}$. Les efforts pour renforcer ce lien entre les partenaires - en particulier ceux qui sont d'âge proche - peuvent poser d'importantes fondations pour faire de la première année de mariage une zone de sécurité ou de santé.

\section{Diminuer la pression imminente pour tomber enceinte}

Peut être que la plus grande difficulté à rendre les rapports sexuels exempts de risque dans le mariage est de trouver des moyens d'augmenter l'acceptabilité du report de la procréation pendant un an au moins alors que le couple est «en train de se connaître » - et il y a encore des doutes sur le statut de VIH. Le cadrage du report de procréation en terme de protection de la fertilité future et d'assurance que les enfants puissent grandir dans une famille stable et saine peut rendre cette approche plus acceptable ${ }^{25}$.

\section{Faire disparaître le stigmate du condom et de la protection contre les IST/VIH dans le mariage}

De nombreuses études ont avancé que le condom était inacceptable dans le mariage, mais

l'augmentation de l'utilisation du condom dans le mariage dans certains endroits réfute ces croyances ${ }^{26}$. Il peut être possible d'augmenter l'utilisation du condom dans le mariage dans certaines cultures si nous reformons l'image du condom pour représenter non la méfiance ou l'infidélité, mais plutôt comme le respect de la santé du partenaire, la protection de sa propre santé et la préservation de la fertilité future. Souligner le lien entre l'infertilité et le VIH peut s'avérer contraignant puisque de nouvelles études ont montré que les femmes VIH positif ont un tiers de chances en moins de concevoir que les femmes VIH négatif $^{27}$.

\section{Premières naissances et au-delà}

Affiner les services de santé maternelle et de santé sexuelle et reproductive des adolescents pour inclure les adolescents mariés dans le cercle

A ce jour, les programmes de santé sexuelle et reproductive des adolescents ont soit ignoré les besoins des adolescentes mariées ou supposé qu'ils étaient identiques à ceux des adolescentes non mariées ${ }^{28}$. De

\footnotetext{
${ }^{23}$ Zulu, Eliya Msiyaphazi, Susan Cotts Watkins, et Gloria Chepngeno. 2002. "Spousal perceptions, comprehension and management of HIV/AIDS risk in rural Malawi," présentation à la Conférence sur le VIH au Malawi faite à l'Université de Pennsylvanie, Octobre

${ }^{24}$ Sundari Ravindran, T.K. 2001. "Programming for low-income married adolescents: An experience from rural Tamil Nadu, India." Genève: OMS, Département du Genre et de la Santé des Femmes

${ }^{25}$ Brady, Martha. 2003. "Differentiating risk perception and protection needs of young women across the marital transition," projet de document présenté à à la Consultation Technique OMS/FNUAP/Population Council sur les Adolescents Mariés, OMS, Genève, 9-12 Décembre

${ }^{26}$ Maharaj, Pranitha and John Cleland. 2003. "The quiet revolution: Condom use within marriage," document de travail présenté à la Conférence Taking Stock of the Condom in the Era of HIV/AIDS, Gaborone, Botswana, 13-17 Juillet; and Clark, Shelley. 2003. "Suspicion, infidelity and HIV among married couples in Malawi," document présenté à la réunion annuelle de l'Association d'Amérique pour la Population, Minneapolis, 1-3 Mai

${ }^{27}$ Glynn, Judith, Anne Buvé, Michael Caraël et al. 2000. "Decreased fertility among HIV-1-infected women attending antenatal clinics in three African cities," Journal of Acquired Immune Deficiency Syndromes 25(4): 345-352

${ }^{28}$ Santhya and Jejeebhoy. 2003. Voir note 15
} 
même, les services de santé maternelle et infantile - le vecteur clé pour l'appui à la santé reproductive des personnes nominalement « adultes »- ont fait des efforts minimaux, lorsqu'ils ont été faits, pour atteindre les plus jeunes mariées (et leurs partenaires). Les services de santé maternelle et infantile qui sont souvent intensifs même dans les pays les plus pauvres pourraient être reconfigurés de sorte à accorder une attention prioritaire aux plus jeunes nouvelles mères. Cette focalisation apporterait une nouvelle énergie significative aux initiatives de maternité sans risque, en ravivant l'intérêt qu'elles suscitent et en les rendant plus pertinentes.

Susciter la prise de conscience du VIH et améliorer la sécurité dans le mariage grâce aux services à la première naissance

L'intervention pendant la première grossesse est justifiée non seulement par le risque objectif qu'elle comporte, mais aussi comme un moment social et psychologique opportun pour poser les fondations pour des habitudes futures positives de santé reproductive. Si ces jeunes femmes n'ont pas encore été atteintes, la première grossesse fournit une opportunité ponctuelle et potentiellement puissante pour dispenser des informations essentielles sur le VIH. L'intervention pendant la première grossesse peut camper le décor pour le futur dialogue et la prise de décision conjointe qui sont cruciaux au bien-être à long terme non seulement des deux époux mais aussi à celui de leurs futurs enfants ${ }^{29}$.

\section{CONCLUSIONS}

Les adolescentes mariées sont en dehors des intérêts de recherche, des diagnostics de politiques et des interventions de base conventionnellement définis (éducation à la vie familiale, centres de jeunes, éducation par les pairs et services de santé conviviaux pour les jeunes) qui ont fixé les programmes de santé reproductive des adolescents ainsi que de nombreuses activités de prévention du VIH/SIDA. Elles sont un segment isolé de la population, souvent important par leur nombre, extrêmement vulnérable, et pour une large part non atteint par les stratégies d'intervention actuelles.

Dans beaucoup de pays, le mariage précoce est une attaque à plusieurs facettes sur les droits des filles et il peut servir de pont pour le virus du VIH vers la population générale. Promouvoir le mariage à une échéance plus tardive, au moins à 18 ans, et soutenir les options de protection, notamment le condom et lorsqu'ils sont disponibles les microbicides ${ }^{30}$ dans le mariage peuvent constituer des moyens essentiels pour endiguer l'épidémie.

Ces mesures pourraient aider le mariage à accomplir son rôle idéalisé comme un havre de protection contre bon nombre des dangers de la vie, et particulièrement le VIH. Afin de rendre le mariage sûr, cependant, nous devons d'abord être disposés à reconnaître une réalité désagréable à savoir que pour beaucoup, il n'est pas sûr. La dénégation des risques du mariage et le maintien des interventions hors de la sphère privée du mariage ne protégeront pas ceux qui sont dans ses liens de la menace croissante du VIH.

\footnotetext{
${ }^{29}$ Graft, Auralice, et al. 2003. "Addressing married adolescents' needs: A review of programs," and Haberland, Nicole, Erica Chong and Hillary Bracken. 2003. "Married adolescents: An overview," projet de document présenté à la Consultation Technique OMS/FNUAP/Population Council sur les Adolescents Mariés, OMS, Genève, 9-12 Décembre

${ }^{30}$ Etant donné la pression et le souhait d'avoir des enfants, les microbicides sont une option particulièrement attrayante. Pour la prévention de la grossesse et de l'infection, le condom (masculin et féminin) est idéal
} 